PF 2019 (LXXIV): 351-363

\author{
GERD HENTSCHEL, SABINE ANDERS-MARNOWSKY, \\ SVIATLANA TESCH \\ Instytut für Slawistik \\ Fakultät III \\ Carl von Ossietzky Universität Oldenburg \\ D-26111 Oldenburg \\ Deutschland \\ tel. +494417984635 \\ e-mail: gerd.hentschel@oldenburg
}

\title{
ZUR RUSSISCHEN VERMITTLUNG VON GERMANISMEN INS POLNISCHE WÄHREND DER ZEIT DER POLNISCHEN TEILUNGEN
}

SCHLÜSSELWÖRTER: lexikalische Entlehnung ins Polnische, Vermittlung von Entlehnungen, Germanismen, Russismen, Polnische Teilungen.

SŁOWA KLUCZOWE: zapożyczenia leksykalne w języku polskim, pośrednictwo zapożyczeń, germanizmy, rusyzmy, rozbiory Polski.

KEYWORDS: lexical borrowing into Polish, mediation of borrowings, Germanisms, Russisms, Partitions of Poland.

\author{
ON THE MEDIATION OF THE RUSSIAN LANGUAGE IN TAKING \\ GERMANISMS INTO POLISH DURING THE PARTITIONS OF POLAND
}

\begin{abstract}
The paper discusses the phenomenon of lexical borrowing of German words into Polish mediated by Russian during the time of the Partitions of Poland from the end of the $18^{\text {th }}$ century to the First World War. It is based on material from an ongoing research project devoted to the question of which path parallel Germanisms took into Polish on the one hand, and into the East Slavic languages on the other, from the late Middle Ages to mid- $20^{\text {th }}$ century: the direct path from German or one mediated by one or more of the corresponding other Slavic languages. It is pointed out that about one fifth of the more than one thousand Germanisms that occurred for the first time in Polish written texts during this time period are accounted for earlier in Russian texts. Though definitely not all of the Germanisms in this set have been mediated by Russian,
\end{abstract}


on the basis of the discussion of seven sample borrowings from the study it is shown that the mediation of lexical material from German via Russian into Polish may be considerably more extensive than has been described so far. Furthermore, it is illustrated that the question of mediation may in principle be addressed by four answers: directly from German, mediated from Russian, unclear, and both, i.e. directly from German a n d mediated by Russian. In the latter case, especially some of several expression variants in Polish reveal a clear affinity with German „originals”, and others with Russian adaptations. In many cases, of course, any answer for an individual Germanism may only be given in terms of the degree of probability.

Der Sprachkontakt zwischen dem Polnischen bzw. Slavischen und dem Deutschen sowie der Kontakt zwischen dem Polnischen und seinen slavischen Nachbarsprachen, nicht zuletzt den ostslavischen, war und ist ein einschlägiges Thema im Schaffen unseres Jubilars. In diese Thematik fallen auch die folgenden Betrachtungen, im Besonderen die Überlagerung der beiden prinzipiellen Kontaktkonstellationen, wie sie sich auch bei deutschen Lehnwörtern im Slavischen zeigt.

Während das Polnische auf das Russische vom Spätmittelalter bis zum 18. Jahrhundert bekanntlich einen deutlichen Einfluss ausgeübt und dabei auch zahlreiche Germanismen ${ }^{1}$ vermittelt hat, so kehrte sich die Richtung der Beeinflussung aufgrund der Umkehrung machtpolitischer Verhältnisse ab der petrinischen Zeit ebenso um, besonders jedoch während der Zeit der polnischen Teilungen. Für die Lexik hat Karaś (1996) den Einfluss des Russischen auf das Polnische der Teilungszeit beschrieben. Unter den von Karaś vermerkten Russismen des Polnischen haben zahlreiche eine deutsche Etymologie. In den folgenden Zeilen soll zumindest angedeutet werden, dass der Umfang der vom Russischen vermittelten Menge von Germanismen noch größer ist, als er von Karaś (1996) dargestellt werden konnte. Im Mittelpunkt steht die Diskussion verschiedener Germanismen der Teilungszeit im Polnischen und Russischen, die in Karaś' Arbeit unerwähnt bleiben oder wenn die dortige Darstellung präzisiert werden kann.

Ausgangspunkt ist dabei das WDLP (2011), das den Anspruch hat, die Germanismen in der polnischen Schrift- bzw. Standardsprache zu beschreiben, die in der Zeit von den Anfängen des polnischen Schrifttums bis in die Mitte des 20. Jhs. direkt aus dem Deutschen entlehnt und nicht etwa vom Tschechischen oder Russischen vermittelt wurden. Fälle, in denen die Frage

1 Die Termini Germanismus, Russismus u. dgl. werden hier eng im Sinne deutscher, russischer Lehnwörter verstanden. Lehnübersetzungen bzw. -übertragungen und nicht lexikalische Transfers bleiben unberücksichtigt. 
nach der Vermittlung der beiden slavischen Sprachen bzw. nach der direkten Entlehnung aus dem Deutschen nicht eindeutig sind, werden im WDLP vielfach diskutiert. Außerdem beschränkt sich dieses (elektronische) Wörterbuch auf Germanismen mit deutscher (germanischer) Etymologie. Elemente, die im Deutschen selbst Lehnwörter sind (vor allem aus dem Latein oder aus romanischen Sprachen), bleiben unberücksichtigt. Ausgehend vom WDLP führt der erstgenannte Autor dieses Beitrags mit Unterstützung der beiden Koautorinnen und dem Institut für deutsche Sprache (IDS) in Mannheim gegenwärtig ein weiteres Forschungsprojekt durch. Dieses trägt den Titel: Wörter auf Wanderschaft: Der Weg deutscher Lehnwörter des Polnischen ins Ostslavische. ${ }^{2}$ In Anlehnung an das WDLP werden in diesem Projekt (kurz mit „DLwPO” angesprochen) grundlegend alle großen historischen und etymologischen Wörterbücher sowie für die Zeit ab dem 19. Jh. die großen zeitgenössischen Wörterbücher ausgewertet: hier natürlich zu den drei ostslavischen Sprachen (HSBM, SBN, TSBM, TSBLM, ĖSBM, SIRJa XI-XVII, SRJaXVIII, TSŽVJa-1, TSŽVJa-4, SSRLJa, TSRuJa, ĖSRJa-F, SStUM XIV-XV, SUM XVI-XVII, ISUJa, MSUM, SUM-Hrin, SUM, VTSSUM, ESUM). Berücksichtigt werden bei den großen historischen Wörterbüchern nicht nur die bisher erschienenen Bände (viele der relevanten Werke sind bisher nicht vollständig erschienen), sondern auch die jeweiligen Karteien ${ }^{3}$.

Will man den Weg der Germanismen vom Polnischen in die ostslavischen Sprachen überprüfen, so gilt es, zunächst „parallele” deutsche Lehnwörter auf beiden Seiten, hier also für das Polnische und für das Russische zu ermitteln. (Das Ukrainische und Weißrussische können meistens ausgeblendet werden, da sie in der hier relevanten Zeit keinerlei vermittelnde Funktion ausübten.) Die Erhebungen des DLwPO ergeben, dass eine sehr große Zahl von parallelen Germanismen im Polnischen (gemäß WDLP) und im Russischen vorliegt, die im Polnischen zwischen 1772 und 1914 zum ersten Mal im Schrifttum anzutreffen sind und im Russischen einen früheren Beleg zeigen. Insgesamt geht es um knapp 200 Lexeme. Diese machen immerhin knapp ein Fünftel aller deutschen Lehnwörter aus, die im WDLP überhaupt mit einem frühesten Beleg in dieser Zeitspanne verzeichnet wurden (vgl. Hentschel 2009). Auch wenn die Arbeiten

2 Dieses Projekt wird von der Deutschen Forschungsgemeinschaft (DFG - Gz. HE 1566/14-1) gefördert. Die Ergebnisse dieses Projekts werden auf dem Lehnwortportal Deutsch des IDS in der Form eines elektronischen Wörterbuchs mit multiplen, komplexen Suchfunktionen zur Verfügung gestellt. Auch dies wird durch die DFG (EN 798/4-1) gefördert - Leitung Stefan Engelberg; Pflege Peter Meyer. Integriert in dieses Portal sind bereits das WDLP und das WDLTDP. (Letzteres ist auf der Basis der Papierausgabe in Siatkowski 2004 besprochen worden.) Der Zugang zum Portal ist unter http://lwp.ids-mannheim.de/ kostenfrei.

3 Wird auf die Karteien dieser Wörterbücher Bezug genommen, so bekommt die Abkürzung des Wörterbuchs die Ergänzung "-K”. 
im DLwPO noch nicht abgeschlossen sind, kann heute schon festgestellt werden, dass die meisten der genannten knapp 200 Lexeme ins Polnische nicht oder sehr wahrscheinlich nicht vom Russischen vermittelt, sondern direkt aus dem Deutschen entlehnt wurden. Diese können hier nicht behandelt werden. Bei knapp einem Viertel sprechen jedoch mal mehr, mal weniger Aspekte dafür, dass das Russische eine vermittelnde Rolle gespielt hat. ${ }^{4}$ Mitunter ist das nur partiell der Fall, d.h. für bestimmte Bedeutungen des Germanismus oder für die ein oder andere Variante der Ausdrucksseite des Wortes im Polnischen. Von letzteren sollen hier einige wenige vorgestellt werden. D.h., dass die Frage nach der russischen Vermittlung nicht nur mit „ja”, „nein” oder „unklar” zu beantworten ist, sondern mitunter auch mit „sowohl als auch”.

fajerwerkarz: fejerwerkier, ab 1810 Lin, Sw (m.u.); fajerwerker, ab 1855 Swil; fejerwerker, ab 1861 Swil; fajerwerkier, ab 1900 Sw; fajerwerkarz, ab 1916 (SPA). Vgl. russ. феирверкер, ab 1711; феиерверкер, ab 1712; фейерверкер, ab ca.1721; феерверкер, ab 1721; фейверкор, ab 1771 - alle im SRJa XVIII; 'ein Artillerieunteroffizier', poln. ab 1855 (Swil, Sw, SPA 1916), russ. ab 1755, sowie 'jmd., der Feuerwerke macht, Pyrotechniker' poln. ab 1810 (Lin, Swil, Sw, SPA 1916 / 1929), russ. ab 1711.

Im Russischen ist das Wort in beiden, schon im Deutschen angelegten Bedeutungen früher belegt als im Polnischen (in der militärischen mit 100 Jahren noch deutlicher als in der zivilen), sodass im Russischen für beide eine direkte Entlehnung aus dem Deutschen anzunehmen ist. (Der früheste Beleg für die zivile Bed. 2 im Russischen stammt aus dem Wörterbuch Novoj leksikon na francuskom [sic], nemeckom, latinskom, i na rossijskom jazykach. (ЛB1)). Auch die früheste Ausdrucksvariante des Polnischen passt perfekt zu den schon ca. 100 Jahre früher belegten russischen, sodass trotz der ersten Erwähnung im Wörterbuch Lindes, der auf den außermilitärischen Bereich (Bed. 2) Bezug nimmt, eine russische Vermittlung nicht nur für den militärischen Kontext (Bed. 1), wie er auch im WDLP und von Karaś (1996: 120) als Möglichkeit angedeutet wird, sondern auch für den außermilitärischen Bereich (Bed. 2) eine hohe Wahrscheinlichkeit hat.

Auffallend ist aber noch folgendes: Dt. Feuerwerk findet sowohl im Russischen als auch im Polnischen in den 90-er Jahren des 17. Jhs. seinen Niederschlag: im Russischen nur mit „e-Diphthong”, im Polnischen auch mit „a-Diphthong”.

4 Ein gutes Viertel der Germanismen mit Erstbelegen im Polnischen während der Teilungszeit und früherem Beleg im Russischen ist im DLwPO noch nicht bearbeitet worden, sodass zu ihnen noch gar keine Einschätzungen vorliegen. Umfassende und sichere quantitative Festlegungen sind ohnehin erst nach Abschluss aller Arbeiten möglich. 
Der russische Erstbeleg geht auf Peter den Großen zurück und dürfte daher auch eher direkt auf das Deutsche zurückgehen, sodass für dt. Feuerwerk eher eine getrennte, parallele Entlehnung anzunehmen ist. Für das Nomen agentis, besonders für die militärische Bedeutung, ist jedoch eine unabhängige spätere Entlehnung mit der dargelegten russischen Vermittlung wahrscheinlicher.

Im Ukrainischen und im Weißrussischen ist nur die militärische Bedeutung nachgewiesen, und zwar erst im 20. Jh. An der Vermittlung des Russischen besteht kein Zweifel.

forszmak: ab 1913 (Sw) - vgl. russ. Форшмак, ab 1833 (SSRLJa - vgl. auch wr. Фаримак; ukr. форшмак, beide erst im 20. Jh.) 'ein Gericht, eine Vorspeise aus gehacktem Hering, Fleisch, Butter, Kartoffeln und Ei'. Gri notiert nhd. Vorschmack 'Vorkost, Aufbiß, Zubiß, auch Frühstück', alternativ zu Vorgeschmack. Der Ausdruck ohne -ge- sowie die Bezeichnung einer Vorspeise ist im Standarddeutschen geschwunden.

Im Standardpolnischen wie in den ostslavischen Literatursprachen liegt das Wort in der zitierten Bedeutung mit Bezug auf ein Gericht mit gehacktem Hering vor. Dies ist eine Bedeutungsverengung: von einer Vorspeise allgemein im Deutschen zu einer bestimmten Vorspeise. Dem Beleg im Russischen aus der ersten Hälfte des 19. Jhs. (SSRLJa s.v. форшмак) steht im Polnischen eine erste Buchung (ohne Beleg) erst im Sw, also am Anfang des 20. Jhs. gegenüber. Vasmer (ÉSRJa-F) geht im Russischen von einer Direktentlehnung aus dem Deutschen aus. Im Ukrainischen ist das Wort ab der ersten Hälfte des 20. Jhs. belegt, im Weißrussischen ist es erst spät im 20. Jh. gebucht. Hier ist jeweils von einer Vermittlung durch das Russische auszugehen. In der kulinarischen Literatur in ostslavischen Sprachen wird форшмак häufig als typisches Gericht der jüdischen Küche beschrieben. (Auf der Ebene der Realien zeigt das Gericht übrigens offenbar eine größere Variation als die lexikographisch erfassten Bedeutungen nahelegen - in allen Sprachen. Dies ist im Internet leicht festzustellen.) Die konsultierten Wörterbücher zum Jiddischen Ast, Stu, Tyšč buchen das Wort jedoch nicht. Im Jiddischen spricht man eher von gehakte herring. In der populären kulinarischen Literatur wird verschiedentlich auf eine Herkunft aus dem Ostpreußischen verwiesen. Das PrWB (Bd. II, 1863) verzeichnet das Wort jedoch nicht. Außerhalb des Kanons der hier ausgewerteten Quellen wird in Hinsicht auf ein erstes Vorkommen für das Russische auf das Kochbuch Russkaja povarnja von V.A. Levšin (Moskva 1816) und für das Polnische auf das von Lucyna Ćwierczakiewiczowa 365 obiadów za 5 złotych (Warszawa 1860) verwiesen. Auch hier deutet sich also ein früherer Nachweis im Russischen an.

Alles spricht somit für eine Übernahme ins Polnische aus dem Osten. Es mag sein, dass die kulinarische Tradition der osteuropäischen Aschkenasim die Realie geprägt hat, von einer Vermittlung des Germanismus durch das Jiddische kann 
angesichts des Fehlens eines entsprechenden jiddischen Ausdrucks aber nicht ausgegangen werden. Da weder die engere Bedeutung im Deutschen nachzuweisen ist, noch das PrWB das Wort verzeichnet, ist ein Übergang des älteren russ. форшмак (mit der entsprechenden Bedeutung) ins Polnische der wahrscheinlichste Weg.

gefrejter: giefrejter, ab 1786 Lin, Swil, Sw; gifrejter, ab 1792 Swil, Sw, Lsp, Dor (daw.); gefrejter, ab 1812 Swil, Lsp, Dor; giefrajter, ab 1913 Sw; gefrajter, ab 1937 Lsp, SZNP. Vgl. russ. гефрейтер, ab 1698; геврейтер, ab 1698; ефрейтор, ab 1705; эфрейтор, ab 1716, ефрейтур, ab 1735; ефрейтер, ab 1742 'Rang zwischen den gemeinen Soldaten und dem Unteroffizier; Gefreiter (im Militär, selten im Polizeidienst)'. Die Bedeutung ist mit der deutschen militärischen Nomenklatur der Dienstränge identisch.

Im Russischen ist das Wort erstmals 1698 in dem Reglement $O$ voen-

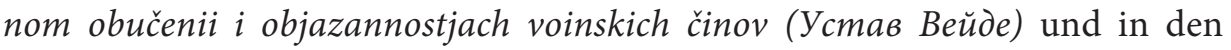
Pis'ma i bumagi imp. Petra Velikogo (ПБП) belegt. Es liegt hier sicherlich eine Direktentlehnung aus dem Deutschen vor. Nach dem 18. Jh. sind im Russischen nur Formen ohne initiales $g$ - nachweisbar. Im Polnischen ist das (heute als veraltet einzustufende) Wort, in der Variante giefrejter (hapax), erstmals 1786 in dem in Warschau gedruckten Regulamen exercerunku Kawaleryi Narodowey (Kaw. Nar.) belegt, also auf russischem Teilungsgebiet. Ebenfalls Ende des 18. Jhs. ist die poln. Var. gifrejter erstmals belegt. Besonders letztere legt eine Übernahme aus dem Russischen nahe. Hätte eine dialektale dt. Aussprache mit j- (je- / ji-) Pate gestanden, so wäre das $g$ - im poln. Anlaut implausibel. Auch das diphthongoide $-e j$ - statt -aj- macht ein russ. Vorbild plausibel, obwohl dt. dialektal auch ein „e-Diphthong” denkbar ist. Seit Anfang des 19. Jhs. tritt die Var. gefrejter auf, erst im 20. Jh. giefrajter und das dem hochsprachlich dt. Muster ähnlichste gefrajter. Der Anlaut ge- stammt sicher aus dem Deutschen, gie- deutet eher auf eine russ. Vorlage hin. Die Geschichte des Wortes im Polnischen spiegelt also in seinen Ausdrucksvarianten sowohl den russischen als auch den deutschen Einfluss wider, was „realiengeschichtlich” naheliegt, da russisch- und deutschsprachige Truppen im Lande standen.

krambambuli: krambambula, ab 1844 Wiecz; krambambuli, ab 1861 Swil, Sw; krampampula, ab 1902 Sw; trambambula, ab 1908 Wiecz; krambamboli, ab 1913 Sw (gw.) - alle subst., f. Vgl. russ. крамбамбули subst., m. indkl., ab 1722, SRJa XVIII, SSRLJa. Nach Gri: dt. Krambambuli 'eine Art süßer Schnaps, zunächst aus Danzig, mit verschiedenen pflanzlichen Zutaten oder ein heißes, süßes Getränk daraus'. Dieselbe Bedeutung liegt auch im Russischen und Polnischen vor.

Das WDLP beschreibt das Wort im Polnischen als direkte Entlehnung aus dem Deutschen. Im Deutschen wird das Wort textuell erst 1747 fassbar, und zwar in Danzig aus einem Trinklied (vgl. Gri s.v.), im Polnischen jedoch erst 
1844 (in Warschau, also im russischen Teilungsgebiet, aber als Femininum auf $-a$ ), anschließend mit beträchtlicher Ausdrucksvariation. Im Russischen ist das Wort nur in der Var. крамбамбули belegt, und zwar genusgleich mit dem dt. Wort, aber erstmals schon 1772 in der in Petersburg erschienenen satirischen Zeitschrift Živopisec, eženedel'noe na 1772 g. sočinenie (Жuв.). Zwar suggeriert Danzig wegen seiner räumlichen Kontingenz eher eine direkte Übernahme ins Polnische, auffallend ist aber neben dem späten Erstbeleg im Polnischen zum einen das Fehlen des Wortes in der Kartei zum SGP und zum anderen sein Fehlen in den drei konsultierten Wörterbüchern zum Jiddischen. (Die Schankwirtschaft im ehemaligen ostpolnischen Raum, heute in Litauen, Weißrussland und in der Ukraine, war bekanntlich weitgehend in jüdischen Händen.) Für das Russische ist angesichts des frühen Belegs und der Genusgleichheit mit dem Deutschen naheliegend, eine direkte Entlehnung aus dem Deutschen anzunehmen. Das besagte deutsche Trinklied wurde dann in den 1830-er Jahren von N.M. Jazykov ins Russische übertragen. Die polnischen Belege sind alle aus der Folgezeit, hauptsächlich aus dem östlichen Raum. Eine Übernahme aus dem Russischen ins Polnische liegt also durchaus näher als eine direkte aus dem Deutschen. Außerdem war und ist das Wort im Russischen offenbar viel weiter verbreitet als im Polnischen: Das Nacionalnyj korpus russkogo jazyka weist Belege für крамбамбули aus der zweiten Hälfte des 20. Jhs. aus - ebenfalls im Liedkontext. Eine Rolle für die Ausweitung (auch in baltische und weißrussische Gebiete, ohne dass wr. Wörterbücher das Wort notieren) könnten studentische Kreise in Petersburg gespielt haben (davor ggf. militärische Kreise und die Seefahrt). Jedenfalls ist die Realie ursprünglich eher eine der Oberschicht als eine des bäuerlichen oder proletarischen Milieus. Eine späte Ausdrucksvariante des Polnischen (krampampula, ab 1902, nur Sw) erinnert merkwürdigerweise durch ihre Lautstruktur an tschechische und slowakische Formen (Newerkla sowie Gri s.v.), wobei hier wie dort laut- oder wortspielerische Momente eine Rolle gespielt haben mögen.

Wenn heute (ab den 2000-er Jahren) in der populären Literatur und im Internet oft krambambuli / krambambulja als weißrussisches Getränk beschrieben wird, so scheint das mit dem Erfolg einer 2001 gegründeten weißrussischen Rockgruppe Krambambulja in Verbindung zu stehen. (Ein weißrussischer Geschäftsmann hat zeitgleich eine entsprechende Spirituose dieses Namens auf den Markt gebracht.) Im weißrussischen Schrifttum und in der weißrussischen Lexikographie gibt es jedoch keine Hinweise auf eine längere Tradition des Wortes.

kurcgalop: ab 1902, Sw, Dor, auch kurs-galop, ab 1913, Sw (beide subst., m.). Vgl. russ. куризгалоп, subst., m., ab 1797, SRJa XVIII, SSRLJa 'kurzer Galopp eines Pferdes; auch übertragen oder ironisch i.S.v. schleppender Gang'. Ein 
Kompositum ist im Deutschen ganz offensichtlich nicht etabliert (im Gegensatz zu z.B. Kreuzgalopp), aber als Ad-hoc-Bildung ausgehend von kurzer Galopp jederzeit möglich.

Im Russischen ist куригалоп erstmals 1797 im Voinskij ustav o polevoj kavalerijskoj službe (УКС) belegt, der während der Regierungszeit Zar Pauls I entstand; es ist eine direkte Entlehnung aus dem Deutschen.

Im Polnischen ist das Wort um 1900 erstmals nachweisbar, in den Varianten kurcgalop, kurs-galop, letzteres wohl aufgrund falscher Analogie zu kurs. Sw verweist auf einen Beleg bei Gabriela Zapolska, die aus dem Gebiet der westlichen Ukraine stammte und außerdem in Krakau, d.h. im österreichischen Teilungsgebiet gearbeitet hat. Verheiratet war sie (wenn auch nicht lange) mit einem Offizier der Zarengarde, der aus litauischem Gebiet, also aus dem russischen Teilungsgebiet stammt wie auch ihre Mutter (aus Warschau). Zapolska kann das Wort also durch deutschen oder russischen Einfluss übernommen haben, was auch für das Polnische generell gilt. Die viel frühere Präsenz des Wortes im Russischen macht - entgegen WDLP - eine Übernahme aus dem Russischen jedoch mindestens ebenso wahrscheinlich.

reda: rejd, subst., m.; ab 1901 Dor, reda, subst., f.; ab 1946 Dor, SZNP. Vgl. russ. peŭda subst., f., ab 1713, SRJa XVIII-K, SSRLJa; ped subst., m., 1715, SRJa XVIII-K; peda subst., f., ab 1715, SRJa XVIII-K; peŭd subst., m., ab 1718, SRJa XVIII-K, TSŽVJa-1, TSŽVJa-4, SSRLJa, TSRuJa; реэдa subst., f., ab 1735, SRJa XVIII-K; 'eine Art Vorhafen, Wasserbereich für Schiffe, die darauf warten, (per Lotse) in den Hafen einlaufen zu können'; vgl. dt. Reede und holl. rede.

Im Polnischen ist das Wort ab Anfang des 20. Jhs. in der Var. rejd bezeugt. Das WDLP nimmt Vermittlung des Russischen an (s. auch Karaś 1996: 139). Nach 1920 deutet sich eine Ersetzung durch reda an, das offenbar eine Neuentlehnung aus dem Deutschen darstellt und die heutige schriftsprachliche Variante ist. Im Russischen liegen Varianten mit monophthongischem -e- offenbar nur im 18. Jh. vor, sodass auf Grund des Zeitabstandes eine russische Vermittlung dieser Formen ans Polnische unwahrscheinlich ist.

Im Russischen ist das Wort in der petrinischen Zeit erstmals belegt, und zwar in den Varianten peйдa (häufig), рейд (häufig), редa (selten), ред (selten), seit 1735 auch in der Var. реэда (selten); bis heute ist nur die Var. peŭd lebendig. Vasmer (ĖSRJa-F s.v. peŭd) nimmt Entlehnung aus dem Niederländischen an; nicht auszuschließen ist jedoch, dass das Wort, zumindest in den Varianten auf red-, parallel aus dem Deutschen entlehnt wurde. Auch ist im Niederdeutschen eine diphthongische Aussprache des Stammvokals möglich. Klu (s.v.) verweist auf diphthongische Varianten sogar im Hochdeutschen (Anfang des 17. Jhs, d.h. früher als die monophthongischen), da das Wort aus dem Niederdeutschen stammt (vgl. auch EWD s.v.). Allein die historischen Umstände machen eine russische 
Entlehnung aus dem Niederländischen (und nicht aus dem Niederdeutschen) wahrscheinlicher.

szyfer: subst., m.; ab 1861. Vgl. russ. шuфep subst., m., ab 1725, SlRJa XI-XVII-K, SRJa XVIII-K, TSŽVJa-1, TSŽVJa-4, SSRLJa, TSRuJa (wr. maøep; ab 1948, ukr. muфeep; ab 1949). (i) 'Schiefergriffel' (nur poln.), (ii) 'Steinart: tonhaltiger Schiefer, der leicht in dünne Platten zu teilen ist und zur Herstellung von Dachziegeln, Schreibtafeln usw. verwendet wird', so im Polnischen und den drei ostslavischen Sprachen, im Polnischen auch in der Bedeutung 'Schiefergriffel, Griffel'.

Die letztgenannte Bedeutung ist mit Gri schon im Deutschen sehr nah an der ursprünglichen Bedeutung von Schiefer, nämlich die eines spitzen, scharfen Splitters, unabhängig vom Material. Daraus hat sich im Deutschen erst die Bezeichnung des Griffels, später die Bezeichnung einer Steinart entwickelt. Den ostslavischen Sprachen fehlt jedoch die Bedeutung 'Griffel'. Genau diese ist aber im Polnischen prominent. Swil, das beide Bedeutungen von szyfer als erstes polnisches Wörterbuch verzeichnet, stellt die Bedeutung des Griffels in den Vordergrund und stellt zur zweiten fest, dass es manchmal anstelle von tupek verwendet würde. Letzteres ist als Bezeichnung der Steinart schon in Lin gebucht.

Die geologische Bedeutung ist im Russischen erstmals bereits 1725 im Werk Kniga o gornom dele bezeugt, einer Übersetzung des 1556 in lateinischer und 1557 in deutscher Sprache erschienenen Werks De re metallica libri XII / Vom Bergkwerck XII Bücher. Der zweitälteste Beleg für das Russische findet sich 1735 in Opisanie ural'skich i sibirskich zavodov (Геннин) des deutsch-russischen Ingenieurs Georg Wilhelm Henning. Im Russischen liegt also zweifellos Direktentlehnung aus dem Deutschen vor, was auch durch die Präsenz von шиферстейн (SRJa XVIII-K) im Russischen des 18. Jhs. unterstrichen wird.

Ukr. шифер und wr. шыфер in derselben Bedeutung sind im 20. Jh. erstmals belegt - sehr wahrscheinlich Übernahmen aus dem Russischen. Eine Vermittlung durch das Polnische ist unwahrscheinlich, da die geologische Bedeutung im Polnischen nur schwach belegt ist und das Auftreten in diesen beiden Sprachen sehr spät ist.

Was die geologische Bedeutung im Polnischen betrifft, so spricht vieles gegen WDLP - für eine Vermittlung durch das Russische. Die ersten Buchungen kommen mit dem Swil (1861) und Sw (1913) aus Wörterbüchern aus dem russischen Teilungsgebiet, nachdem das Wort im Russischen schon anderthalb Jahrhunderte nachweisbar ist. Ein Einfluss des Deutschen kann natürlich nicht völlig ausgeschlossen werden. 
Die wenigen hier zu diskutierenden Germanismen des Polnischen zeigen somit alle einen mehr oder weniger ausgeprägten Einfluss des Russischen, auf teils unterschiedliche Art und Weise. In jedem der hier diskutierten Fälle - das sei wiederholt - sind die Nachweise im Russischen erheblich früher. Im Falle von poln. forszmak und krambambuli ist russische Vermittlung der naheliegende Weg ins Polnische (gegen oder eher gegen die bisherige Lehrmeinung). Ausschlaggebend sind hier nicht zuletzt sprachexterne, kultur- und sozialhistorische Argumente. Im Falle von fajerwerkarz, gefrejter und reda spielen deutlich sowohl das Russische als auch das Deutsche eine Rolle. Festzumachen ist das hauptsächlich an den Ausdrucksvarianten im Polnischen. In allen Fällen sind die eher ans Russische erinnernden Formen die früheren, im Falle von fajerwerkarz jedoch zeitlich nicht allzu distant. Besonders bei reda zeichnet sich ab, dass Ausdrucksvarianten mit einer Affinität zum Deutschen sich später etablieren, nachdem zuvor solche mit Ähnlichkeit zum Russischen dominiert hatten. Bei szyfer ist für eine der beiden Bedeutungen von einer direkten Übernahme aus dem Deutschen auszugehen, bei der zweiten spricht dagegen vieles für eine russische Vermittlung. Und bei kurcgalop schließlich ist beides in etwa mit derselben Wahrscheinlichkeit möglich.

Die hier vorgestellten sieben Wortskizzen deuten das Spektrum der Komplexität der Frage nach dem Weg von Germanismen ins Polnische der Teilungszeit an. Polen stand unter der Herrschaft zweier deutschsprachiger Mächte und des russischen Zarenreiches, dessen Sprache selbst unter starkem deutschen Einfluss stand. Die Auswahl der hier besprochenen Wörter ist zum Teil dem Zufall des Fortschrittes der Arbeiten am laufenden Projekt des DLwOP geschuldet. Dabei war ein Kriterium, dass die Elemente eine gewisse Verbreitung im Polnischen hatten und partiell noch haben. Für Germanismen des Polnischen der Teilungszeit gilt, wie in Hentschel (2009) bereits dargelegt, dass ein großer Teil der sehr großen Zahl an neuen Germanismen sehr kurzlebig war, und zwar ohne dass in Polen besonders starke puristische Tendenzen festzustellen sind (anders als z.B. im Tschechischen - vgl. Thomas 1991: 43 et passim). Der deutsch-polnische Sprachkontakt war in dieser Zeit in seinen lexikalischen Spuren sehr extensiv, viel weniger intensiv (nachhaltig) - und darüber hinaus z.T. nur indirekt, d.h. vom Russischen vermittelt. Viele, auch der oben angesprochenen 200, früher im Russischen als im Polnischen jener Zeit belegten Germanismen werden nur im Sw gebucht, teils mit, teils ohne Verweise auf Primärquellen. Allerdings sind es bei den nur im Sw gebuchten Germanismen mit lautlicher und / oder semantischer Affinität zu solchen des Russischen wohl weniger die dialektologischen Interessen von Jan Karłowicz (das SGPKarł erschien in zeitlicher Überlappung mit dem Sw), als die Herkunft aller drei Redakteure des Sw aus dem russischen Teilungsgebiet. Darüber hinaus hatte Karłowicz bekanntlich fünf Jahre in Moskau studiert. 
Wir hoffen, im DLwPO, illustriert an den oben dargebotenen kurzen Wortgeschichten, durch einen systematischen Vergleich von Ausdrucks- und Inhaltsstrukturen, von Datierungen im Polnischen und Russischen (sowie im Weißrussischen und Ukrainischen) und nicht zuletzt unter Berücksichtigung der kultur- und soziohistorischen Hintergründe einen umfassenden Überblick über die parallelen Germanismen in den genannten slavischen Sprachen bieten zu können, über ihre gemeinsamen und getrennten Wege.

\section{Bibliographie}

\section{Wörterbücher}

Ast - Аляксандар Астравух, Ідыш-беларускі слоўнік, Мінск 2008.

DLwPO - Wörter auf Wanderschaft: Der Weg deutscher Lehnwörter des Polnischen ins Ostslavische (In Vorbereitung).

Dor - Słownik języka polskiego, red. Witold Doroszewski, t. 1-11, Warszawa 1958-1969. ĖSBM - Этымалагічны слоўнік беларускай мовы, Мінск 1978 -.

ÉSRJa-F - Макс Фасмер, Этимологический словарь русского языка, Москва 19861987.

ESUM - Етімологічний словник української мови, Київ 1982-2012.

EWD - Etymologisches Wörterbuch des Deutschen, Berlin 1989.

Gri - Deutsches Wörterbuch von Jacob und Wilhelm Grimm. 16 Bde. in 32 Teilbänden.

Leipzig 1854-1961 (Quellenverzeichnis Leipzig 1971).

HSBM - Гістарычны слоўнік беларускай мовы, Мінск 1982 -.

ISUJa - Євген Тимченко, Історичний словник українського язика, Харків-Київ 1930-1932, cf. http://litopys.org.ua/djvu/tymchenko_slovnyk.htm [11.12.2018].

Klu - Friedrich Kluge, Etymologisches Wörterbuch der deutschen Sprache, Berlin ${ }^{25}$ 2011.

Lin - Samuel B. Linde, Słownik języka polskiego, t. 1-6, Warszawa 1807-1814.

Lsp - Tadeusz Lehr-Spławiński, Słownik języka polskiego, Warszawa 1935-1939.

MSUM - Василь B. Німчук, Галина I. Лиса, Євген Тимченко, Матеріали до слов-

ника писемної та книжної української мови XV-XVIII ст. Київ-Нью-Йорк 2002. PrWB - Hermann Frischbier, Preußisches Wörterbuch. Ost- und westpreußische

Provinzionalismen in alphabetischer Folge, Bd. I-II. Berlin 1882/1883.

SBN - Иван И. Носович, Словарь белорусского наречия, Санкт-Петербург 1870.

SGP - Słownik gwar polskich, 1977 i n., opr. przez Zakład Dialektologii Polskiej Instytutu

Języka Polskiego PAN w Krakowie, pod red. Mieczysława Karasia, od t. II pod red.

Jerzego Reichana, od t. VI pod red. Joanny Okoniowej, od t. IX pod kier. Renaty

Kucharzyk, Wrocław-Kraków.

SGPKarł - Jan Karłowicz, Słownik gwar polskich, t. 1-6, Warszawa 1900-1911.

SIRJa XI-XVII - Словарь русского языка XI-XVII вв., Москва 1975 -.

SPA - Słownik ilustrowany języka polskiego Michała Arcta, Warszawa 1916 / 1929. 
SRJaXVIII - Словарь русского языка XVIII в., Ленинград / Санкт-Петербург 1984 i n., cf. http://feb-web.ru/feb/sl18/slov-abc/ [1.12.2108].

SSRLJa - Словарь современного русского литературного языка, т. 1-17, Москва 1948-1965.

SStUM - Словник староукраїнської мови XIV-XV ст., Київ 1977-1978.

Stu - Nachum Stutchkoff, Der Oytser Fun Der Yiddisher Sprakh, New York 1950,

cf. http://www.cs.uky.edu/ raphael/yiddish/searchOytser.cgi [1.12.2108].

SUM - Словник української мови, ред. Іван К. Білодід, т. I-XI, Київ 1970-1980.

SUM XVI-XVII - Словник української мови XVI - першої половини XVII ст., Львів $1994-$.

SUM-Hrin - Борис Грінченко Словарь української мови, т. I-IV, Київ 1907-1909.

Sw - Słownik języka polskiego, red. Jan Karłowicz, Adam A. Kryński, Władysław

Niedźwiedzki, t. I-VIII, Warszawa 1900-1927.

Swil - Słownik języka polskiego, red. Aleksander Zdanowicz et al., t. 1-2, Wilno 1861.

SZNP - Marek Łaziński, Słownik zapożyczeń niemieckich w polszczyźnie, Warszawa 2008.

TSBLM - Тлумачальны слоўнік літаратурнай беларускай мовы, Мінск 2005.

TSBM - Тлумачальны слоўнік беларускай мовы, т. 1-5, Мінск 1977-1984.

TSRuJa - Толковый словарь русского языка, ред. Сергей И. Ожегов, Наталия Ю. Шведова, Москва 2013.

TSŽVJa-1 - Владимир И. Даль, Толковый словарь живаго великорускаго языка, Москва 1863.

TSŽVJa-4 - Иван А. Бодуэн-де-Куртенэ, Толковый словарь живого великорусскаго языка Владиміра Даля, С.-Петербург-Москва 1912-1918 (4. изд.).

Туг̌с̌ - Дмитро В. Тищенко, Їдиш-український словник, ред. О. Бейдерман, С. Вашецька, М. Потольський, Київ 2016.

VTSSUM - Великий тлумачний словник сучасної української мови, Київ 2009.

WDLP - Andrzej de Vincenz, Gerd Hentschel, Wörterbuch der deutschen Lehnwörter in der polnischen Schrift- und Standardsprache: Von den Anfängen des polnischen Schrifttums bis in die Mitte des 20. Jahrhunderts, Studia Slavica Oldenburgensia. Oldenburg 2010, http://www.bis.uni-oldenburg.de/bis-verlag/wdlp/ [1.12.2018].

Wiecz - Bronisław Wieczorkiewicz, Słownik gwary warszawskiej XIX w., Warszawa 1966.

\section{Anderes}

Hentschel Gerd, 2009, Intensität und Extensität deutsch-polnischer Sprachkontakte von den mittelalterlichen Anfängen bis ins 20. Jahrhundert am Beispiel deutscher Lehnwörter im Polnischen. In: Christel Stolz (Hrg.) Unsere sprachlichen Nachbarn in Europa. Die Kontaktbeziehungen zwischen Deutsch und seinen Grenznachbarn. (Diversitas Linguarum), Bochum, 155-171.

Karaś Halina, 1996, Rusycyzmy słownikowe w polszczyźnie okresu zaborów, Warszawa. Siatkowski Janusz, 2004, rez. T. Menzel, G. Hentschel, Wörterbuch der deutschen Lehnwörter im Teschener Dialekt des Polnischen, Oldenburg 2003. Zeitschrift für slavische Philologie, Bd. 63/1 (2004), 232-237.

Thomas George, 1991, Linguistic purism. London. 


\section{Streszczenie}

Artykuł poświęcony jest fenomenowi przenikania germanizmów za pośrednictwem rosyjskiego do polskiego. Na wybranych przykładach autor pokazuje, że liczba w ten sposób zapożyczanych germanizmów jest najprawdopodobniej większa niż dotąd zakładano. Przy systematycznym uwzględnianiu wariantów danego słowa na poziomie wyrażenia oraz polisemii pojawiają się często układy, w których określone warianty wyrażenia i/ lub znaczenia wskazują na pośrednictwo rosyjskiego w procesie zapożyczania, niektóre natomiast bezpośrednio $\mathrm{z}$ niemieckiego trafiły do polszczyzny. 